\title{
Modeling the Kelvin polarization force actuation of Micro- and Nanomechanical systems
}

\author{
Schmid, Silvan; Hierold, C.; Boisen, Anja
}

Published in:

Journal of Applied Physics

Link to article, DOI:

10.1063/1.3309027

Publication date:

2010

Document Version

Publisher's PDF, also known as Version of record

Link back to DTU Orbit

Citation (APA):

Schmid, S., Hierold, C., \& Boisen, A. (2010). Modeling the Kelvin polarization force actuation of Micro- and Nanomechanical systems. Journal of Applied Physics, 107(5). https://doi.org/10.1063/1.3309027

\section{General rights}

Copyright and moral rights for the publications made accessible in the public portal are retained by the authors and/or other copyright owners and it is a condition of accessing publications that users recognise and abide by the legal requirements associated with these rights.

- Users may download and print one copy of any publication from the public portal for the purpose of private study or research.

- You may not further distribute the material or use it for any profit-making activity or commercial gain

- You may freely distribute the URL identifying the publication in the public portal 


\title{
Modeling the Kelvin polarization force actuation of micro- and nanomechanical systems
}

\author{
Silvan Schmid, ${ }^{1, a)}$ Christofer Hierold $^{2}$ and Anja Boisen ${ }^{1}$ \\ ${ }^{1}$ Department of Micro- and Nanotechnology, Technical University of Denmark, DTU Nanotech, \\ Building 345 East, DK-2800 Kongens Lyngby, Denmark \\ ${ }^{2}$ Department of Mechanical and Process Engineering, Micro and Nanosystems, ETH Zurich, 8092 Zürich, \\ Switzerland
}

(Received 20 November 2009; accepted 7 January 2010; published online 9 March 2010)

Polarization forces have become of high interest in micro- and nanomechanical systems. In this paper, an analytical model for a transduction scheme based on the Kelvin polarization force is presented. A dielectric beam is actuated by placing it over the gap of two coplanar electrodes. Finite element method simulations are used to characterize the scheme and to evaluate a field correction factor, which results from simplifying the form of the electric field. The model has been shown to be valid for dielectrics with different permittivities. The presented model facilitates the design of microresonators and nanoresonators with dielectric actuation, which offers a great freedom in the choice of structural material. (c) 2010 American Institute of Physics. [doi:10.1063/1.3309027]

\section{INTRODUCTION}

Polarization forces have been widely used to manipulate dielectric objects. With an inhomogeneous electric field dielectric particles can be manipulated, which is known as dielectrophoresis. ${ }^{1-3}$ With it, small solid spherical particles can be levitated in dielectric medium. ${ }^{4,5}$ It is also often used for cell manipulation and separation. ${ }^{6,7}$ The dielectrophoretic force has been used to make contact less tweezers for single cell manipulation. ${ }^{8}$ Furthermore, it has been used to control biomolecules, ${ }^{9}$ DNA,${ }^{10,11}$ nanowires, ${ }^{12}$ bundles of carbon nanotubes ${ }^{13}$ and single-walled carbon nanotubes. ${ }^{14}$

Polarization forces are also used in electrohydrodynamic continuous flow micropumps ${ }^{15,16}$ and to operate dropletbased lab-on-a-chip. ${ }^{17}$

Inspired by a talk of Feynman ${ }^{18}$ a scheme for a dielectric nanomotor was elaborated. ${ }^{19}$ Dielectric micromotors for the application in a liquid medium made from polyimide have been designed and tested. ${ }^{20}$ An electrostatic glass actuator working in air has also been realized. ${ }^{21}$

It has been shown that polarization forces are very interesting for the actuation of micro- and nanomechanical structures. A dielectric microbeam or nanobeam is placed over the gap of two coplanar electrodes. The beam is then attracted toward the electrode gap, that is toward the electric field intensity maximum. This force is called Kelvin polarization force or dielectric force. Based on this actuation scheme, dielectric microresonators made from polymeric materials have been successfully actuated in vacuum ${ }^{22}$ and in air and water. ${ }^{23}$ Lately, the dielectric force has also been applied in nanomechanical systems actuating silicon nitride strings. ${ }^{24}$ The movement of the dielectric beam induces a change of the capacitance over the two electrodes, which can be used for the readout of the beam movement. ${ }^{24,25}$ Because this actuation scheme does not need an electrical connection to the dielectric resonator, in principle the actuation and readout

${ }^{a)}$ Electronic mail: silvan.schmid@nanotech.dtu.dk. can be decoupled from the resonator itself. This can be of interest for disposable resonant sensors where the sophisticated and expensive actuation and readout unit is kept for many measurements and the dielectric resonator unit is exchanged for every measurement. This actuation scheme does not impose constraints on the choice of the structural material as e.g., piezoelectrical actuation. The resonators can be built from one pure material and does not need any metalization, which could increase the damping. ${ }^{26,27}$ Because of the purity of the structural material such resonators are well suited for material characterization. ${ }^{28,29}$

There are basically two principle designs with which a microresonator or nanoresonator based on the Kelvin polarization force can be realized. The electrode configuration can be parallel (parallel plate capacitor) or coplanar and the force on the dielectric is directed parallel to the electrodes or normal to the electrodes, respectively. An analytical model for the dielectric slab moving parallel in between the electrodes of a parallel plate capacitor has been derived and tested by means of the finite element method (FEM) ${ }^{22}$ In this work, the force acting on a dielectric that is placed over two coplanar electrodes (Fig. 1) is modeled. A dielectric beam is attracted toward the highest electric field intensity over the gap of the two coplanar electrodes on which a potential difference is applied. This design which has already been

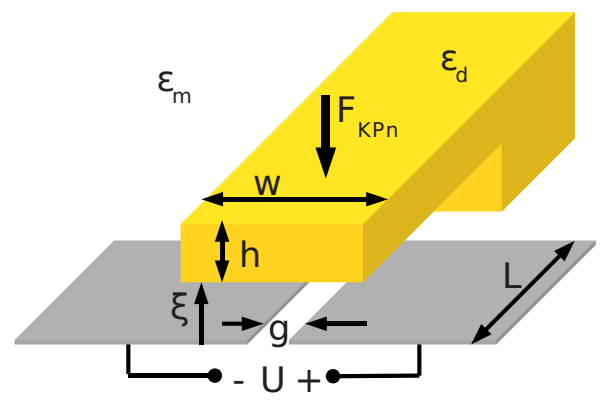

FIG. 1. (Color online) Actuation scheme of a dielectric beam experiencing the Kelvin polarization force normal $\left(F_{\mathrm{KPn}}\right)$ toward the electrodes. 
applied $^{22,24}$ has the advantage that it can be fabricated by means of surface micromachining. The actuation scheme for dielectric microresonators and nanoresonators based on the Kelvin polarization force has the potential for many applications. In this paper, a simple analytical model for an actuator design based on polarization forces is derived.

\section{POLARIZATION FORCES IN DIELECTRIC MATERIALS}

The most common force in context with dielectrics is the dielectrophoretic force. Dielectrophoresis is defined as the translational motion of neutral matter caused by polarization effects in a nonuniform electric field. ${ }^{1}$ Pohl $^{30}$ coined this term to distinguish between this effect and electrophoresis, which requires charged particles. The polarization which is responsible for the dielectrophoretic force is defined by the properties of the neutral object to be moved. The force resulting from the polarization of the dielectric material is called the Kelvin polarization or dielectric force. The Kelvin polarization force is defined by the bulk material properties of the involved dielectrics.

The term dielectrophoresis is closely related to the movement of neutral particles in a liquid. Therefore, the single particles are treated as individual dipoles, assuming that the contribution of the dipoles to the electric field $\mathbf{E}$ is zero. The force on an infinitesimal dipole can then be approximized by ${ }^{2}$

$$
\mathbf{F}=\mathbf{p} \cdot \nabla \mathbf{E},
$$

where $\mathbf{p}$ is the dipole moment. From Eq. (1) it is evident that an electric field has to be inhomogeneous in order to exert a net force on a dipole. For bulkier objects that cannot be simplified as an individual dipole, the polarization force acting on the object can be calculated by means of the polarization bulk force. From a microscopic view, the infinitesimal dipoles situated in an electric field experience a force that they pass on to the medium as a whole. With the macroscopic polarization $\mathbf{P}$, the Kelvin polarization force density ${ }^{31}$ is obtained

$$
\mathbf{f}_{\mathrm{KP}}=\mathbf{P} \cdot \nabla \mathbf{E} \text {. }
$$

With the polarization of a linear dielectric material with a susceptibility $\chi_{\mathrm{e}}$

$$
\mathbf{P}=\varepsilon_{0} \chi_{\mathrm{e}} \mathbf{E}=\varepsilon_{0}\left(\varepsilon_{\mathrm{r}}-1\right) \mathbf{E},
$$

the Kelvin polarization force density can be written as ${ }^{31}$

$$
\mathbf{f}_{\mathrm{KP}}=\frac{1}{2} \varepsilon_{0}\left(\varepsilon_{\mathrm{r}}-1\right) \nabla(\mathbf{E} \cdot \mathbf{E}) .
$$

Equation (4) depicts that the strength of the force depends on the polarizability and the volume of the attracted medium and the field intensity squared.

If a dielectric object with a dielectric constant $\varepsilon_{\mathrm{d}}$ is surrounded by a dielectric medium with $\varepsilon_{\mathrm{m}}$, it has been shown that the polarization in Eq. (3) can be replaced by the excess polarization per unit volume ${ }^{32}$

$$
\mathbf{P}_{\mathrm{e}}=\varepsilon_{0}\left(\varepsilon_{\mathrm{d}}-\varepsilon_{\mathrm{m}}\right) \mathbf{E} .
$$

Thus, the Kelvin polarization force acting on a dielectric object with volume $V$ surrounded by a dielectric medium becomes

$$
\mathbf{F}_{\mathrm{KP}}=\int_{V} \frac{1}{2} \varepsilon_{0}\left(\varepsilon_{\mathrm{d}}-\varepsilon_{\mathrm{m}}\right) \nabla(\mathbf{E} \cdot \mathbf{E}) \mathrm{d} V .
$$

In an aqueous environment, however, charges, bound ions (Stern layer), and a diffuse double layer create electric shells at the interface between the object and the water. ${ }^{33}$ These surface layers have a distinct dielectric behavior which is important in dielectrophoresis. 3,34,35 These induced charges alter the net polarization of a dielectric object. This effect is not treated in this work but has to be considered in an application where a dielectric is surrounded by water. Furthermore, the permittivities of dielectric materials are frequency dependent. There are different dielectric mechanisms, such as dipole orientation, atomic polarization, and electronic polarization, which contribute to the dielectric behavior of a material. Each mechanism has a characteristic cut-off frequency at which it is too slow to follow the alternating electric field. The dipolar water molecules, for example, are highly polarizable at low frequencies. But at around $20 \mathrm{GHz}$, the dielectric constant of water decreases rapidly. ${ }^{36}$ However, dielectrics without dipolar mechanisms have a permittivity that is constant upto the terahertz region. ${ }^{37}$ Thus, for dielectrics with atomic and electronic mechanisms only, the Kelvin polarization force stays constant upto this frequency region.

The Kelvin polarization force density sufferes the weakness that the interaction between dipoles is not taken into account. In order to overcome this shortcoming, an approach based on the principle of virtual work introduced by Korteweg and Helmholtz is applied. ${ }^{38}$ Assuming the electrical constitutive law takes the form of a state function

$$
\mathbf{E}=\mathbf{E}\left(\alpha_{1} \ldots \alpha_{\mathrm{m}}, \mathbf{D}\right),
$$

where the $\alpha$ 's are the material properties and $\mathbf{D}$ is the electric displacement. The application of the principle of virtual work allows the deduction of the electric force density with combined effects of free charge and polarization. This generalized form also called the Korteweg-Helmholtz polarization force density is ${ }^{38}$

$$
\mathbf{f}_{\mathrm{KHP}}=\rho_{\mathrm{f}} \mathbf{E}-\sum_{i=1}^{\mathrm{m}} \alpha_{i} \nabla\left(\frac{\partial W}{\partial \alpha_{i}}\right),
$$

where $\rho_{\mathrm{f}}$ is the free charge density and $W$ is the stored electrical energy given by

$$
W=\int_{0}^{\mathbf{D}} \mathbf{E}\left(\alpha_{1} \ldots \alpha_{\mathrm{m}}, \mathbf{D}^{\prime}\right) \cdot \mathrm{d} \mathbf{D}^{\prime} .
$$

Because the Korteweg-Helmholtz polarization force density has been derived with a thermodynamic technique it includes the interaction between dipoles which was not taken into account in the derivation of the Kelvin polarization force.

If the constitutive law takes the form of an electrically linear material 


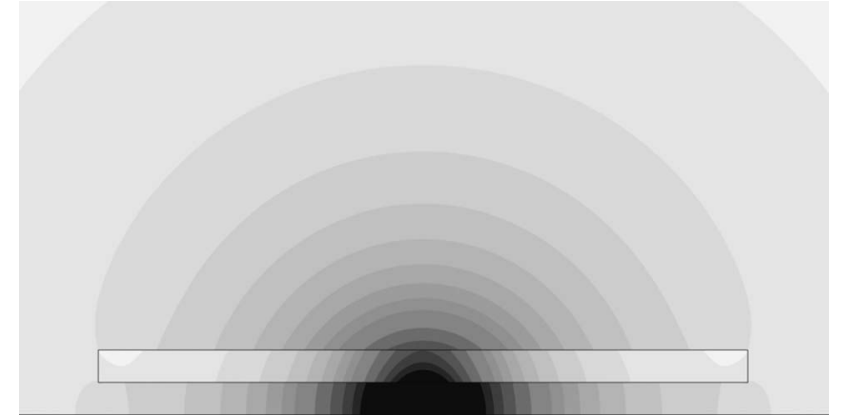

(a)

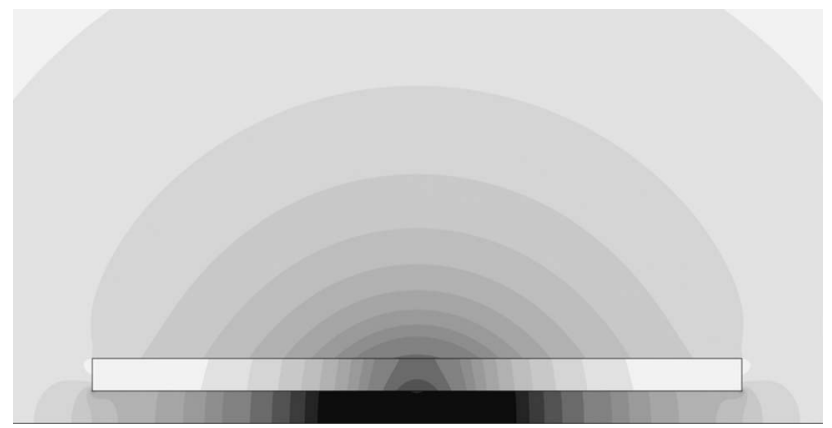

(b)

FIG. 2. Plot of the electric field over two electrode plates disturbed by a dielectric slab $(w=20 \mu \mathrm{m}$ and $h=1 \mu \mathrm{m})$ surrounded by vacuum for a dielectric constant of $\varepsilon_{\mathrm{d}}=2$ (a) and $\varepsilon_{\mathrm{d}}=7$ (b). The electric field was simulated with FEM (Comsol).

$$
\mathbf{D}=\varepsilon_{0}\left(1+\chi_{e}\right) \mathbf{E}
$$

and $\alpha_{1}$ is taken as $\chi_{\mathrm{e}}$, the stored electrical energy and its derivative becomes

$$
W=\frac{1}{2} \frac{D^{2}}{\varepsilon_{0}\left(1+\chi_{\mathrm{e}}\right)} ; \quad \frac{\partial W}{\partial \chi_{\mathrm{e}}}=-\frac{1}{2} \varepsilon_{0} E^{2} .
$$

To obtain this result it is important that the field $\mathbf{E}$ is only changed by changing $\alpha$ 's and not $\mathbf{D}$. With Eq. (11) the force density specializes to

$$
\mathbf{f}_{\mathrm{KHP}}=\rho_{\mathrm{f}} \mathbf{E}+\frac{1}{2} \varepsilon_{0} \chi_{\mathrm{e}} \nabla E^{2} .
$$

Assuming that there is no free charge $\rho_{\mathrm{f}}$, the KortewegHelmholtz polarization force density for a linear dielectric in Eq. (12) is equal to the Kelvin polarization force density in Eq. (4).

\section{ANALYTICAL MODEL}

When the dielectric beam is introduced into the electric field it will be polarized and in return it alters the field. At the boundary between the dielectric with $\varepsilon_{\mathrm{d}}$ and the surrounding medium with $\varepsilon_{\mathrm{m}}$ a polarization surface charge is induced by the field. If $\varepsilon_{\mathrm{d}}>\varepsilon_{\mathrm{m}}$ the charge weakens the field inside the dielectric relative to the field of the surrounding medium and for $\varepsilon_{\mathrm{d}}<\varepsilon_{\mathrm{m}}$ the field inside the dielectric is strengthened. In Fig. 2, a simulation of the electric field is shown for dielectric beams with two different relative dielectric constants. It can be seen, that the field inside the beam is only slightly changed for a small $\varepsilon_{\mathrm{d}}$ and it is still concentric as an approxi-

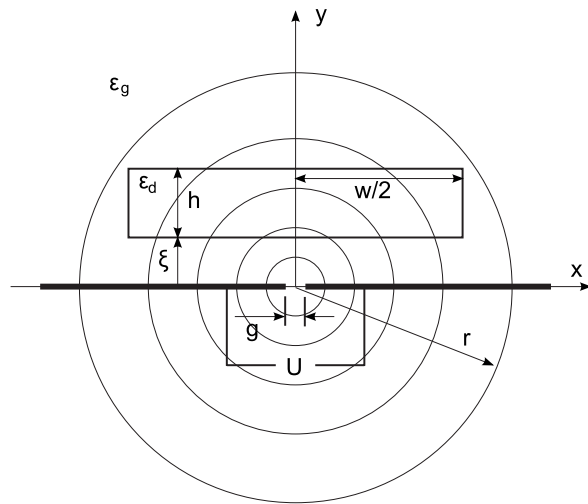

FIG. 3. Schematic drawing of the electric field for the modeling of the $F_{\mathrm{KPn}}$.

mation. Theoretically, the electric field can be calculated by solving Laplace's equation in each region of constant permittivity and using Gauss's and Faraday's constitutive laws as the jump conditions at the surfaces of discontinuity between the dielectric beam and the dielectric medium. ${ }^{31}$ In this work, as an approximation, the electric field is assumed to be concentric over the whole area. Figure 3 depicts the actuator design and the corresponding idealistic electric field. Assuming the electrode gap $g$ to be infinitesimal small, the electric field for a voltage $U$ produced by the two electrodes without the presence of the dielectric is written as

$$
E_{0}=\frac{U}{\pi r}=\frac{U}{\pi \sqrt{x^{2}+y^{2}}}
$$

The assumption of having a perfectly concentric electric field is only valid for the hypothetical case when the dielectric beam has the same permittivity as the medium $\left(\varepsilon_{\mathrm{d}}=\varepsilon_{\mathrm{m}}\right)$. In that case the polarization force is zero, as a matter of course. However, if the permittivities are not equal, the concentric electric field in the medium is disturbed by the dielectric beam with the consequence, that the field is not perfectly concentric both, in the beam and in the medium. As already mentioned above, the field strength inside the dielectric beam is a function of the difference in permittivity of the beam and the surrounding medium. Therefore, the factor $\alpha$ is introduced. The field inside the dielectric beam is now written as

$$
E_{\mathrm{d}}=\alpha E_{0},
$$

where $\alpha$ represents the field reduction and field imperfection inside the dielectric material. With Eq. (4), the Kelvin polarization force density in the normal direction to the electrodes inside the dielectric is

$$
f_{\mathrm{KPn}}=\frac{1}{2} \varepsilon_{0}\left(\varepsilon_{\mathrm{d}}-\varepsilon_{\mathrm{m}}\right) \frac{\partial E_{\mathrm{d}}^{2}}{\partial y}=-\varepsilon_{0}\left(\varepsilon_{\mathrm{d}}-\varepsilon_{\mathrm{m}}\right) \frac{\alpha^{2} U^{2} y}{\pi^{2}\left(x^{2}+y^{2}\right)^{2}} \text {. }
$$

To obtain the force acting on the dielectric beam, the force density has to be integrated over its volume. 


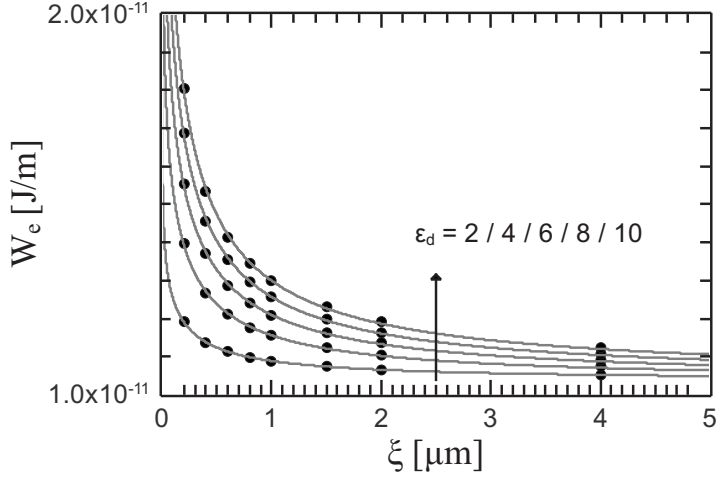

FIG. 4. Fits of Eq. (18) to the field energy calculated with FEM for dielectric beams with $h=600 \mathrm{~nm}$ and $w=20 \mu \mathrm{m}$ surrounded by air or vacuum $\left(\varepsilon_{\mathrm{m}}=1\right)$. The electrode gap was $g=0.1 \mu \mathrm{m}$ and the voltage $U=1 \mathrm{~V}$.

$$
\begin{aligned}
F_{\mathrm{KPn}}(\xi)= & L \int_{\xi}^{\xi+h} \int_{-w / 2}^{w / 2} f_{\mathrm{KPn}} \mathrm{d} x \mathrm{~d} y=\frac{1}{\pi^{2}} \varepsilon_{0}\left(\varepsilon_{\mathrm{d}}\right. \\
& \left.-\varepsilon_{\mathrm{m}}\right) \alpha^{2} U^{2} L \cdot \frac{\xi \arctan \frac{w}{2(\xi+h)}-(\xi+h) \arctan \frac{w}{2 \xi}}{\xi(\xi+h)} .
\end{aligned}
$$

Assuming the beam width to be large compared to its height and its distance to the electrodes, the force can be simplified to

$$
F_{\mathrm{KPn}}(\xi)=-\frac{1}{2 \pi} \varepsilon_{0}\left(\varepsilon_{\mathrm{d}}-\varepsilon_{\mathrm{m}}\right) \alpha^{2} L \frac{h}{\xi(\xi+h)} U^{2}
$$

One can see that in vacuum or air, the $F_{\mathrm{KPn}}$ is negative, that means it acts toward the electrodes. If the dielectric is surrounded by water which has a very high permittivity of $\left(\varepsilon_{\mathrm{m}} \approx 80\right)$ the force is positive and the beam is pushed away from the electrodes. To finally use this expression to define the polarization force, the weighting factor $\alpha$ has to be known.

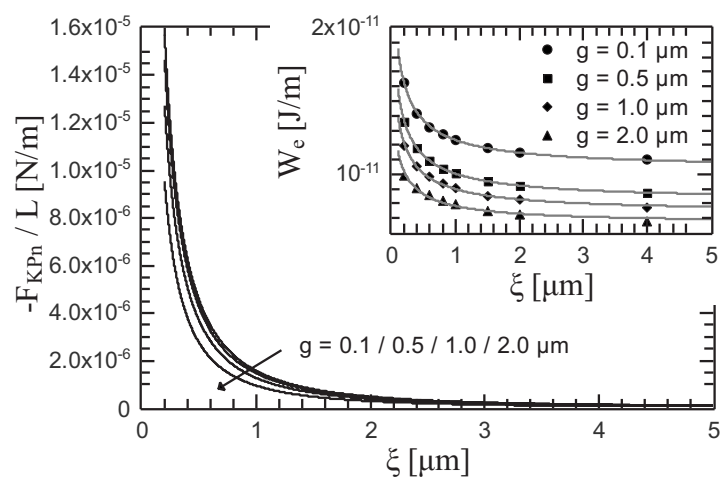

FIG. 5. $F_{\mathrm{KPn}}$ plotted against the distance $\xi$ of the dielectric with $w$ $=40 \mu \mathrm{m}, h=600 \mathrm{~nm}$, and $\varepsilon_{\mathrm{d}}=7$ from the coplanar electrodes with an applied potential of $U=1 \mathrm{~V}$ in air or vacuum $\left(\varepsilon_{\mathrm{m}}=1\right)$. The four curves represent $F_{\mathrm{KPn}}$ for different electrode gap sizes $g$. The insert shows the stored electrical field energy $W_{\mathrm{e}}$ simulated with FEM. The simulated values are fitted with Eq. (18) with the fitting parameter $\alpha$. The fit was of high quality with coefficients of determination $R^{2}>0.999$.

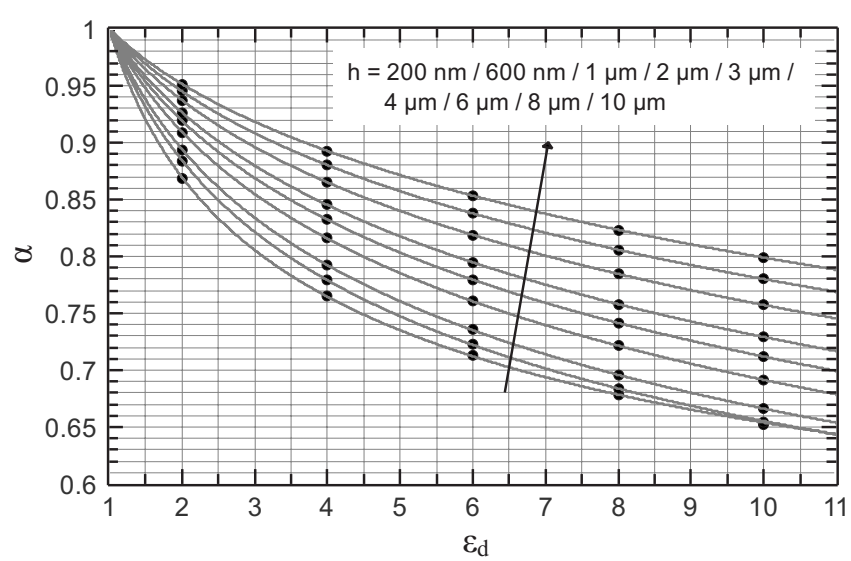

FIG. 6. $\alpha$ plotted for different dielectric constants and heights. The FEM simulations were performed with the following conditions: $g=0.1 \mu \mathrm{m}, w$ $=60 \mu, \varepsilon_{\mathrm{m}}=1$, and $U=1 \mathrm{~V}$. The lines are heuristic fits corresponding to Eq. (19).

\section{FEM}

In this section, the analytical model is tested with FEM and the field correction factor $\alpha$ is extracted.

In order to evaluate the analytical model it is compared to the force simulated with FEM. Therefore, the electric field is simulated and the electric energy density is integrated over the total area of the model. The electrical energy in the system is given by

$$
\begin{aligned}
W_{\mathrm{e}}(\xi) & =-\int F_{\mathrm{KPn}}(\xi) \mathrm{d} \xi \\
& =c-\varepsilon_{0}\left(\varepsilon_{\mathrm{d}}-\varepsilon_{\mathrm{m}}\right) \alpha^{2} U^{2} L \frac{1}{2 \pi} \cdot[\ln (\xi)-\ln (\xi+h)],
\end{aligned}
$$

where $c$ is an integration constant. The simulated values of the electrical energy as a function of the distance $\xi$ can now be fitted with Eq. (18) and so the factor $\alpha$ can be determined.

First, the assumption of having a perfect concentric field is tested for beams with different permittivities. Therefore, the analytical model [Eq. (18)] is fitted to the field energy calculated with FEM for beams with varying dielectric constants (see Fig. 4).

Next, the influence of the electrode gap distance $g$ on the Kelvin polarization force is evaluated. Figure 5 shows the polarization force in air or vacuum calculated by means of the FEM. The force was determined for different electrode gap sizes.

Figure 6 shows the values for $\alpha$ for dielectric beams with different dielectric constants $\varepsilon_{\mathrm{d}}$ and heights $h$. The values are obtained by fitting the model [Eq. (18)] to the energy data calculated with FEM.

In Fig. 7, the force acting on dielectric beams with different widths is evaluated for varying electrode gaps $g$ and varying distances $\xi$.

\section{DISCUSSION}

Assumptions and simplifications have been made in order to obtain a model describing the polarization force for 

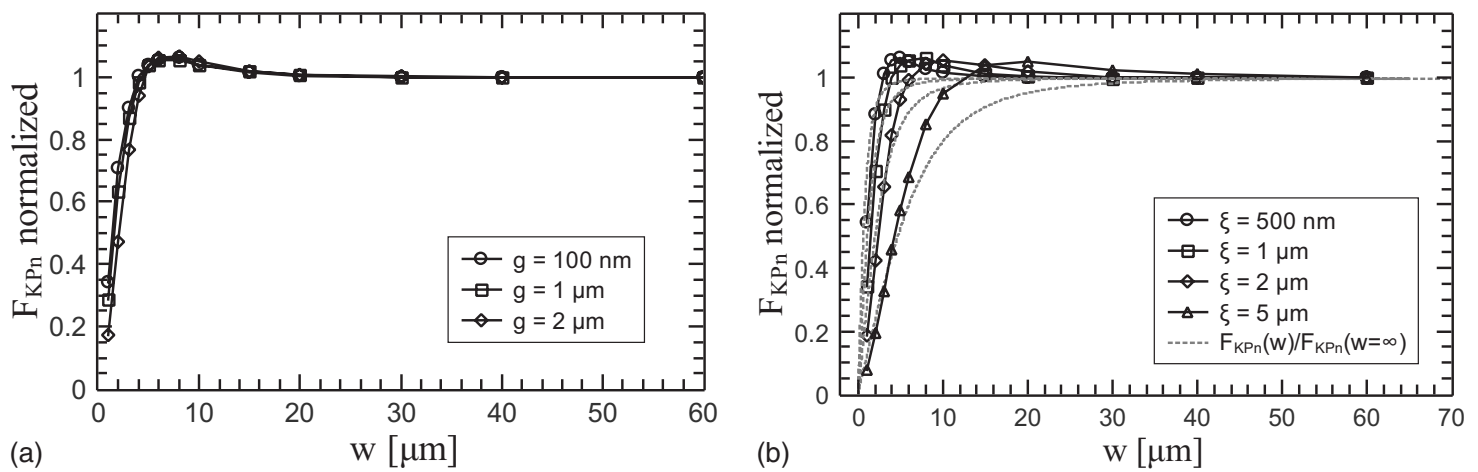

FIG. 7. Normalized $F_{\mathrm{KPn}}$ plotted for beams with $h=600 \mathrm{~nm}$ and $\varepsilon_{\mathrm{r}}=7$ with different widths $w$ for a constant distance $\xi=1 \mu \mathrm{m}$ and a varying electrode gap (a) and for a constant electrode gap $g=100 \mathrm{~nm}$ and a varying distance $\xi(\mathrm{b})$. The FEM simulations were done with the following parameters: $\varepsilon_{\mathrm{m}}=1$ and $U=1 \mathrm{~V}$. The gray dotted lines are plots of Eq. (21) for the corresponding parameters. The black lines are a guide to the eye.

the transduction scheme. Among others, in the derivation of the analytical model it has been assumed that the electric field is concentric even though this is only the case if $\varepsilon_{\mathrm{d}}$ $=\varepsilon_{\mathrm{m}}$. In Fig. 4, it can be seen that the energy values obtained with different dielectric constants $\varepsilon_{\mathrm{d}}$ can well be described by the model [Eq. (18)]. The fits are of high quality with coefficients of determination $R^{2}>0.999$. Thus, the characteristic of the polarization force $F_{\mathrm{KPn}}$ for a wide dielectric beam can be described by assuming a concentric electric field.

In a real transduction scheme, the electrode gap has a finite value ranging from submicrons in case of defining the electrodes with e-beam lithography to one or two microns for conventional photolithography. Even though the gap size has a strong influence on the magnitude of the field energy it has only little influence on the force. In the inset of Fig. 5, it can be seen, that the simulated field energy values can be well described by the analytical model [Eq. (18)]. The force slowly starts to decrease for electrode gaps $g>2 \mu \mathrm{m}$. Consequently, the electrode gap should be designed to be as small as possible but it is not a crucial parameter.

In Fig. 6, the field correction values are shown as a function of $\varepsilon_{\mathrm{d}}$ and $h$. The polarization force acting on a dielectric beam surrounded by air or vacuum $\left(\varepsilon_{\mathrm{m}} \approx 1\right)$ moving perpendicular toward coplanar electrodes can thus be calculated with Eq. (17) together with the specific value for $\alpha$ given in Fig. 6. $\alpha$ can either be read out from Fig. 6 or it can be calculated from the following heuristic formula

$$
\alpha=\frac{8301 h^{0.6156}}{8301 h^{0.6156}+\left(\varepsilon_{\mathrm{d}}\right)^{b}-1},
$$

with

$$
b=0.5887-\frac{0.003795}{h^{0.3184}} .
$$

With this equation, the simulated values for $\alpha$ can be approximized with an average relative error of $2.0 \%$.

In the model, it has been assumed that the width $w$ is large compared to the distance $\xi$. In Fig. 7, it can be seen that from a certain beam width, the force does not become stronger. Thus, the assumption is justified in most cases. In Fig. 7(a) it is shown, that the gap distance $g$ has almost no influence on the force even when the width of the beam approaches the dimensions of the electrode gap. Whereas in
Fig. 7(b) it is shown that the force starts to decrease when the beam width approaches the dimension of the distance $\xi$. The normalized force can be described by dividing Eq. (16) by Eq. (17) which results in

$$
\frac{F_{\mathrm{KPn}}(w)}{F_{\mathrm{KPn}}(w=\infty)}=\frac{2}{\pi h}\left[(h+\xi) \arctan \frac{w}{2 \xi}-\xi \arctan \frac{w}{2(h+\xi)}\right] .
$$

The analytical solution [Eq. (21)] describes the overall force decrease well. But it misses the force maximum just before the force diminishes as given by the simulated normalized force values. Thus, in order to obtain a maximum beam deflection, the beam width should be chosen to be just wide enough that the force is maximal. That is, it should be a few times wider than the distance to the electrodes.

Comparing $F_{\mathrm{KPn}}$ to the normal force acting on parallel capacitor plates ${ }^{39}$

$$
F_{\mathrm{el}}=-\frac{1}{2} \varepsilon_{0} \varepsilon_{\mathrm{m}} w L \frac{1}{\xi^{2}} U^{2},
$$

shows that the two forces are similar. Both are of electrostatic nature and proportional to the applied voltage squared. The main difference is that $F_{\mathrm{el}}$ is a linear function of the beam width whereas $F_{\mathrm{KPn}}$ is a function of the beam height. Increasing the beam height strongly increases the flexural rigidity of the beam and is thus not an option to increase the polarization force strength. As a result, $F_{\text {el }}$ is typically one order of magnitude higher than the polarization force.

Water has a very high polarizability $\left(\varepsilon_{g}=80\right)$ and the area with the field intensity maximum is preferably occupied by the water. So, the electric field intensity close to the gap and therewith the total energy stored in the electric field is minimized. Consequently, the force becomes positive corresponding to Eq. (17) and the dielectric beam experiences a force away from the electrodes. In an aqueous environment, however, polarization forces are highly influenced by surface charges, as already mentioned in Sec. II. The specific behavior of the accumulated interface charges depends on the interaction between the dielectric material and the aqueous solution and the dissolved ions in the solution. Consequently, the exact force acting on a dielectric beam immersed in water cannot be described by the Kelvin polarization force only. 
Nevertheless, based on the polarization body force, the force strength in water is around one order of magnitude higher than in air or vacuum. This is due to the large difference between the permittivity of water and the permittivity of a typical dielectric material with $\varepsilon_{\mathrm{d}}<10$.

\section{CONCLUSION}

In summary, an analytical model describing the force acting in micro- and nanomechanical systems that are based on the Kelvin polarization force has been developed. The model has a simple form and is easy to apply. The force can be calculated with Eq. (17) together with the correction factor $\alpha$ that can either be read out from Fig. 6 or calculated from the heuristic formula (19). Even though polarization forces are considerably weaker than conventional electrostatic forces, they are suitable to actuate dielectric microresonators and nanoresonators made of arbitrary dielectric materials. The presented model offers a simple and fast way to calculate the forces being exerted on the dielectric structures which is important for the design of systems based on this dielectric transduction scheme, which allows the full exploration and employment of specific material properties of pure dielectric materials in micro- and nanomechanical systems.

\section{ACKNOWLEDGMENTS}

The authors would like to thank Søren Dohn from DTU Nanotech for the helpful discussions. This work has been financed by the EU FP7 NANODEVICE project.

${ }^{1}$ H. A. Pohl, Dielectrophoresis (Cambridge University Press, Cambridge, 1978).

${ }^{2}$ T. B. Jones, Electromechanics of Particles (Cambridge Universtiy Press, Cambridge, 1995).

${ }^{3}$ P. R. C. Gascoyne and J. Vykoukal, Electrophoresis 23, 1973 (2002).

${ }^{4}$ G. A. Kallio and T. B. Jones, IEEE Trans. Ind. Appl. IA-16, 69 (1980).

${ }^{5}$ L. Qian, M. Scott, K. V. I. S. Kaler, and R. Paul, J. Electrost. 55, 65 (2002).

${ }^{6} \mathrm{H}$. Li and R. Bashir, Sens. Actuators B 86, 215 (2002).

${ }^{7}$ J. Voldman, Annu. Rev. Biomed. Eng. 8, 425 (2006).

${ }^{8}$ T. P. Hunt and R. M. Westervelt, Biomed. Microdevices 8, 227 (2006)

${ }^{9}$ A. P. Weber, M. Seipenbusch, and G. Kasper, J. Nanopart. Res. 5, 293 (2003).

${ }^{10}$ J. Regtmeier, T. T. Duong, R. Eichhorn, D. Anselmetti, and A. Ros, Anal. Chem. 79, 3925 (2007).
${ }^{11}$ S. Tuukkanen, A. Kuzyk, J. J. Toppari, V. P. Hytönen, T. Ihalainen, and P. Törmä, Appl. Phys. Lett. 87, 183102 (2005).

${ }^{12}$ P. A. Smith, C. D. Nordquist, T. N. Jackson, T. S. Mayer, B. R. Martin, J. Mbindyo, and T. E. Mallouk, Appl. Phys. Lett. 77, 1399 (2000).

${ }^{13}$ C. K. M. Fung, V. T. S. Wong, R. H. M. Chan, and W. J. Li, IEEE Trans. Nanotechnol. 3, 395 (2004).

${ }^{14}$ B. R. Burg, J. Scheider, M. Muoth, L. Durrer, T. Helbling, N. C. Schirmer, T. Schwamb, C. Hierold, and D. Poulikakos, Langmuir 25, 7778 (2009).

${ }^{15}$ D. J. Laser and J. G. Santiago, J. Micromech. Microeng. 14, R35 (2004).

${ }^{16} \mathrm{P}$. Woias, Sens. Actuators B 105, 28 (2005).

${ }^{17}$ J. Zeng and T. Korsmeyer, Lab Chip 4, 265 (2004).

${ }^{18}$ R. Feynman, J. Microelectromech. Syst. 2, 4 (1993).

${ }^{19}$ M. P. Hughes, Nanotechnology 13, 157 (2002).

${ }^{20}$ R. Hagedorn, G. Fuhr, T. Müller, T. Schnelle, U. Schnakenberg, and B. Wagner, J. Electrost. 33, 159 (1994).

${ }^{21}$ R. Moser, R. Wüthrich, L. Sache, T. Higuchi, and H. Bleuler, J. Electrost. 55, 97 (2002).

${ }^{22}$ S. Schmid, M. Wendlandt, D. Junker, and C. Hierold, Appl. Phys. Lett. 89, 163506 (2006)

${ }^{23}$ S. Schmid, P. Senn, and C. Hierold, Sens. Actuators, A 145-146, 442 (2008).

${ }^{24}$ Q. P. Unterreithmeier, E. M. Weig, and J. P. Kotthaus, Nature (London) 458, 1001 (2009).

${ }^{25}$ S. Schmid, Electrostatically Actuated All-Polymer Microbream Resonators-Characterization and Application. Scientific Reports on Micro and Nanosystems (Der Andere Verlag, Tönning, Germany, 2009), Vol. 6.

${ }^{26}$ R. Sandberg, K. Mølhave, A. Boisen, and W. Svendsen, J. Micromech. Microeng. 15, 2249 (2005).

${ }^{27}$ L. Sekaric, D. W. Carr, S. Evoy, J. M. Parpia, and H. G. Craighead, Sens. Actuators, A 101, 215 (2002).

${ }^{28}$ S. Schmid and C. Hierold, J. Appl. Phys. 104, 093516 (2008).

${ }^{29}$ S. Schmid, S. Kühne, and C. Hierold, J. Micromech. Microeng. 19, 065018 (2009).

${ }^{30}$ H. A. Pohl, J. Appl. Phys. 29, 1182 (1958).

${ }^{31}$ H. A. Haus and J. R. Melcher, Electromagnetic Fields and Energy (Prentice-Hall, Englewood Cliffs, NJ, 1989), Chap. 11.

${ }^{32}$ H. A. Pohl, K. Pollock, and J. S. Crane, J. Biol. Phys. 6, 133 (1978).

${ }^{33}$ J. N. Israelachvili, Intermolecular and Surface Forces (Academic Press, New York, 1991).pp.480.

${ }^{34}$ M. P. Hughes and N. G. Green, J. Colloid Interface Sci. 250, 266 (2002).

${ }^{35}$ A. Castellanos, A. Ramos, A. Gonzales, N. G. Green, and H. Morgan, J. Phys. D 36, 2584 (2003).

${ }^{36}$ J. Barthel, K. Bachhuber, R. Buchner, and H. Hetzenauer, Chem. Phys. Lett. 165, 369 (1990).

${ }^{37}$ Basics of Measuring the Dielectric Properties of Materials (Agilent Technologies, Santa Clara, 2006).

${ }^{38}$ J. R. Melcher, Continuum Electromechanics (MIT Press, Cambridge, MA, 1981).

${ }^{39}$ M. Bao, Analysis and Design Principles of MEMS Devices (Elsevier, New York, 2005), pp. 176. 